\title{
Gold-Coated M13 Bacteriophage as a Template for Glucose Oxidase Biofuel Cells with Direct Electron Transfer
}

\author{
RITA A. BLAIK, ESTHER LAN, YU HUANG, and BRUCE DUNN* \\ UCLA, HSSEAS School of Engineering \& Applied Sciences \\ Department of Materials Science and Engineering \\ 410 Westwood Plaza \\ 3111 Engineering V \\ Los Angeles, CA 90095-1595
}

\section{SUPPLEMENTAL INFORMATION}

UV-Vis absorption of native GOx was used to generate a calibration curve by which to determine the concentration of the prepared apoGOx (Figure S1). Figure S2 shows the phage affinity to a gold substrate by a topographical AFM scan before and after EDC-NHS attachment of the gold nanoparticles. Samples were prepared by exposing the phage to the substrate followed by rinsing to remove any unattached phage.

The weight percentage of gold on the phage/gold electrode was calculated using image analysis of the HRTEM data shown in Figure 1c. The average size of the gold nanoparticles is $1.4 \mathrm{~nm}$, and the average size of the phage is $880 \times 6.6 \mathrm{~nm}$. Therefore, the number of phage in the area measured was determined by the width of the strands in the image. The weight of the gold nanoparticles was calculated by determining the amount of gold in an average gold nanoparticle. The weight of the phage is approximately 16.3MDa(1). ImageJ software was used to calculate the number of gold nanoparticles in a given area, which was then extrapolated to the entire phage surface.

Based on the calculated ratio of the surface area of the phage ( 900x6.5nm) and the average surface size of one side of GOx ( $6 \times 5 \times 8 \mathrm{~nm})$, geometric considerations suggest that each phage can accommodate nearly 600 enzymes. Based on the amount of enzymes used in the preparation of the electrode, this gives a final enzyme surface coverage on the phage of about $45 \%$, or $\sim 270$ enzymes per phage.

Overall, our phage/gold/recGOx electrode gives a competitive performance to other reported GOx DET systems using various materials (Table S1). 


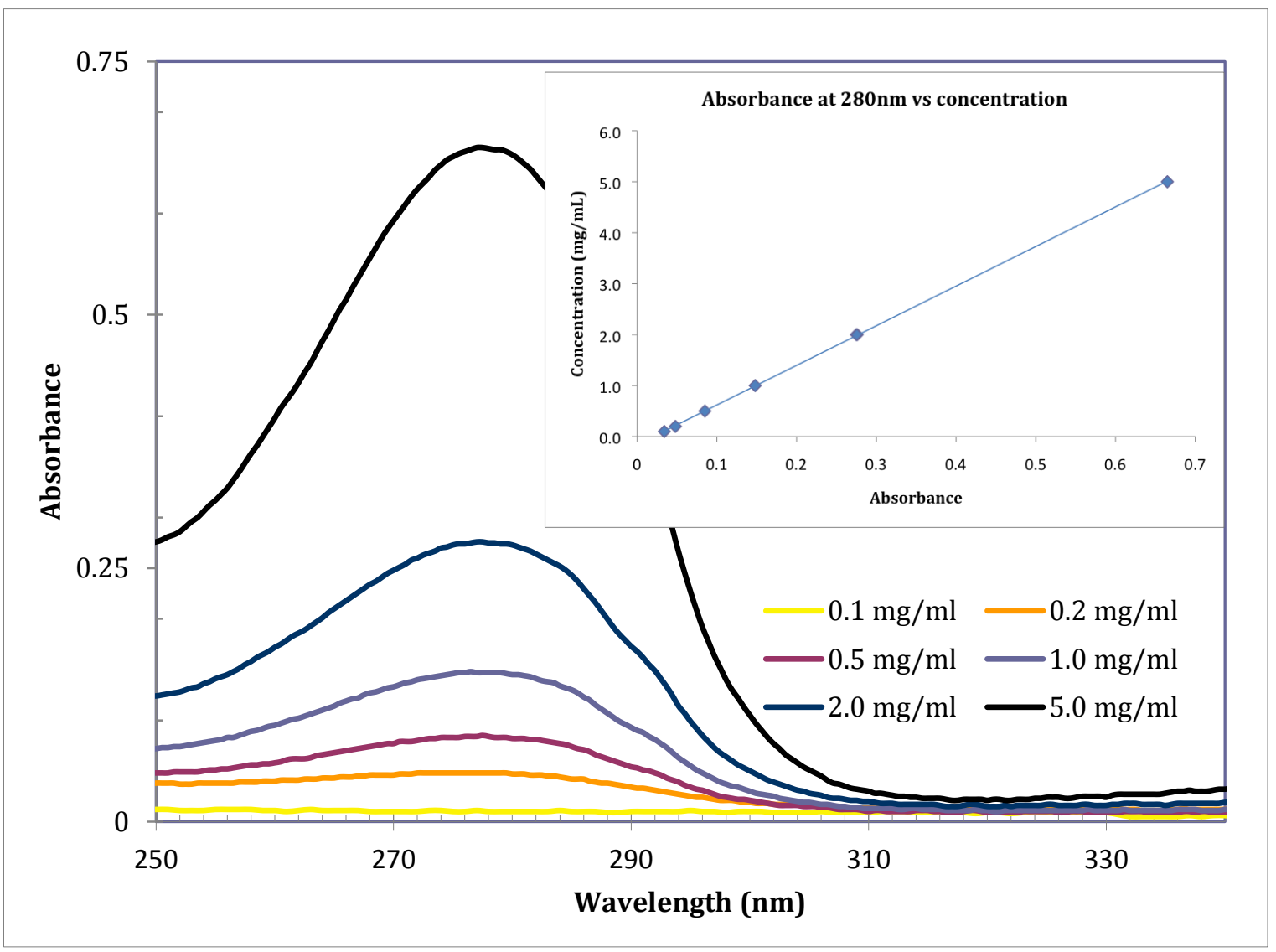

Figure S1. UV-Vis absorption spectrum and calibration curve generated from native GOx in $\mathrm{pH} 5.5$ phosphate buffer to use as a standard by which to determine apoGOx concentration.
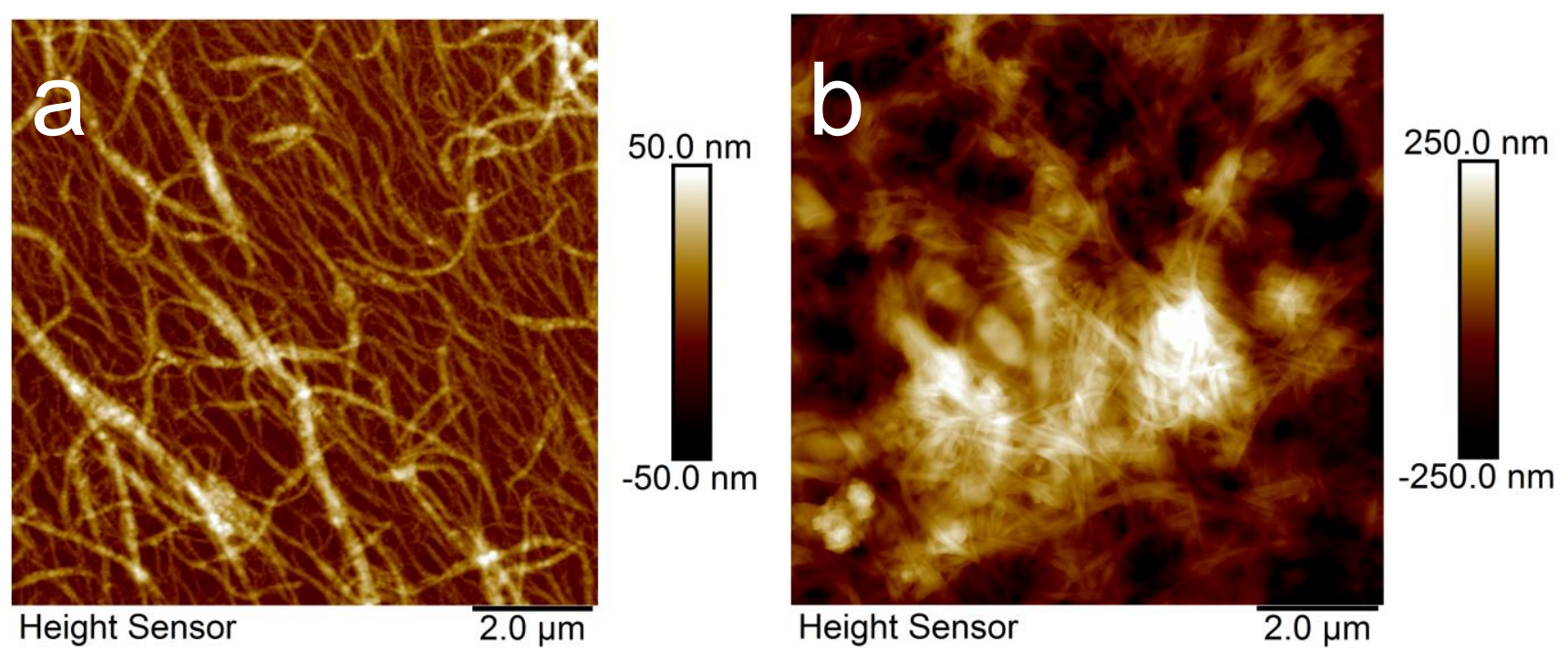

Figure S2. AFM showing phage affinity to a gold substrate before (a) and after (b) EDC-NHS chemistry. Samples were prepared by exposing the phage to the substrate for 1 hour followed by rinsing with deionized water to remove the unattached phage. The scale bar to the right shows the z-axis (top/down) absolute value from the starting point of the needle before it crosses the area being measured. 


\begin{tabular}{|c|c|c|c|c|c|}
\hline $\begin{array}{l}\text { Material, Attachment } \\
\text { scheme }\end{array}$ & $\begin{array}{l}\text { Scan } \\
\text { rate } \\
(\mathrm{mV} / \mathrm{s})\end{array}$ & $\begin{array}{l}\text { Glucose } \\
\text { concentration } \\
(\mathrm{mM})\end{array}$ & $\begin{array}{l}\text { Enzyme } \\
\text { Surface } \\
\text { Coverage }\end{array}$ & $\begin{array}{l}\text { Peak current per } \\
\text { area }\left(\mathrm{mA} / \mathrm{cm}^{2}\right), \\
\text { Voltage }(\mathrm{V})\end{array}$ & Ref. \\
\hline $\begin{array}{l}\text { This work (Phage/gold, } \\
\text { covalent by EDC-NHS) }\end{array}$ & 100 & 50 & $\begin{array}{l}4.74 \times 10^{-8} \\
\mathrm{~mol} / \mathrm{cm}^{2}\end{array}$ & $1.2,0.7$ & ---- \\
\hline $\begin{array}{l}\text { rGO/AuNp composite, } \\
\text { adsorbed }\end{array}$ & 110 & 90 & unknown & $1.5,0.17$ & 2 \\
\hline $\begin{array}{l}\mathrm{PQQ} / \text { Gold, covalent } \\
\text { coupling by soaking }\end{array}$ & 5 & 80 & $\begin{array}{l}1.7 \times 10^{-12} \\
\mathrm{~mol} / \mathrm{cm}^{2}\end{array}$ & $1,0.1$ & 3 \\
\hline $\begin{array}{l}\text { CNT/Carbon paper, } \\
\text { adsorbed }\end{array}$ & 100 & 20 & $\begin{array}{l}0.7 \times 10^{-12} \\
\mathrm{~mol} / \mathrm{cm}^{2}\end{array}$ & $0.5,0.5$ & 4 \\
\hline $\begin{array}{l}\text { Hydroxyl fullerenes } \\
\text { modified glassy carbon, } \\
\text { adsorbed }\end{array}$ & 50 & unknown & $\begin{array}{l}8.3 \times 10^{-11} \\
\mathrm{~mol} / \mathrm{cm}^{2}\end{array}$ & $0.18,-0.4$ & 5 \\
\hline $\mathrm{rGO} / \mathrm{CNT}$ dispersion & 10 & 150 & unknown & $1.4 \times 10^{-2},-0.44$ & 6 \\
\hline $\begin{array}{l}\text { Modified glassy carbon, } \\
\text { adsorbed then EDC-NHS }\end{array}$ & 100 & 10 & unknown & $8.6 \times 10^{-2},-0.5$ & 7 \\
\hline $\begin{array}{l}\mathrm{MoS}_{2} \text { nanosheets/AuNp } \\
\text { modified glassy carbon, } \\
\text { adsorbed }\end{array}$ & 100 & unknown & $\begin{array}{l}2.41 \times 10^{-12} \\
\mathrm{~mol} / \mathrm{cm}^{2}\end{array}$ & $7.9 \times 10^{-2},-0.45$ & 8 \\
\hline nanoporous $\mathrm{TiO}_{2} / \mathrm{CNT}$ & 10 & 5 & unknown & $6.5 \times 10^{-2}, 0.8$ & 9 \\
\hline CNT, adsorbed & 50 & 25 & unknown & $5.7 \times 10^{-2},-0.35$ & 10 \\
\hline $\begin{array}{l}\text { poly(L-arginine)/CNT, } \\
\text { adsorbed }\end{array}$ & 50 & 10 & $\begin{array}{l}1.76 \times 10^{-10} \\
\mathrm{~mol} / \mathrm{cm}^{2}\end{array}$ & $3.9 \times 10^{-2},-0.4$ & 11 \\
\hline $\begin{array}{l}\text { poly(2,6- } \\
\text { diaminopyridine)/CNT, } \\
\text { covalent glutaraldehyde } \\
\text { linker }\end{array}$ & 100 & 8 & unknown & $3.3 \times 10^{-2},-0.4$ & 12 \\
\hline $\begin{array}{l}\text { AuNP modified } \\
\text { graphene/CNT, } \\
\text { electrostatic attraction }\end{array}$ & 50 & 2 & $\begin{array}{l}2.22 \times 10^{-10} \\
\mathrm{~mol} / \mathrm{cm}^{2}\end{array}$ & unknown area & 13 \\
\hline $\begin{array}{l}\text { Mesocellular graphene } \\
\text { foam, adsorbed }\end{array}$ & 50 & 12 & $\begin{array}{l}4.5 \times 10^{-11} \\
\mathrm{~mol} / \mathrm{cm}^{2}\end{array}$ & unknown area & 14 \\
\hline
\end{tabular}

Table S1. Expanded comparison of peak current values and enzyme concentration for different GOx DET schemes taken with cyclic voltammetry. When not directly given, peak current per area was calculated by taking the peak current for the stated CV conditions and dividing by the stated surface area of the electrode. Not all reported systems reached peak current saturation or were scanned within the same voltage range. Enzyme surface coverage values given are calculated by the authors of each reference. 


\section{REFERENCES}

1. Armstrong, N.; Adey, N. B.; McConnell, S. J.; Kay, B. K. Phage Display of Peptides and Proteins: A Laboratory Manual. Kay, B. K.; Winter, J.; McCafferty, J., Eds. Academic Press: San Diego, 1996; pp 35-53.

2. Das, D.; Ghosh, S.; Basumallick, I. Electrochemical Studies on Glucose Oxidation in an Enzymatic Fuel Cell with Enzyme Immobilized on to Reduced Graphene Oxide Surface. Electroanalysis 2014, 26, 2408-2418.

3. Katz, E.; Riklin, A.; Heleg-Shabtai, V.; Willner, I.; Buckmann, A. F. Glucose Oxidase Electrodes via Reconstitution of the ApoEnzyme: Tailoring of Novel Glucose Biosensors. Analytica Chimica Acta 1999, 385, 45-58.

4. Ivnitski, D.; Branch, B.; Atanassov, P.; Apblett, C. Glucose Oxidase Anode for Biofuel Cell Based on Direct Electron Transfer. Electrochem Commun 2006, 8, 1204-1210.

5. $\quad$ Gao, Y. F.; Yang, T.; Yang, X. L.; Zhang, Y. S.; Xiao, B. L.; Hong, J.; Sheibani, N.; Ghourchian, H.; Hong, T.; Moosavi-Movahedi, A. A. Direct Electrochemistry of Glucose Oxidase and Glucose Biosensing on a Hydroxyl Fullerenes Modified Glassy Carbon Electrode. Biosens Bioelectron 2014, 60, 30-34.

6. $\quad$ Grosse, W.; Champavert, J.; Gambhir, S.; Wallace, G. G.; Moulton, S. E. Aqueous Dispersions of Reduced Graphene Oxide and Multi Wall Carbon Nanotubes for Enhanced Glucose Oxidase Bioelectrode Performance. Carbon 2013, 61, 467-475.

7. Liu, G. Z.; Paddon-Row, M. N.; Gooding, J. J. A Molecular Wire Modified Glassy Carbon Electrode for Achieving Direct Electron Transfer to Native Glucose Oxidase. Electrochem Commun 2007, 9, 2218-2223.

$8 . \quad$ Su, S.; Sun, H. F.; Xu, F.; Yuwen, L. H.; Fan, C. H.; Wang, L. H. Direct Electrochemistry of Glucose Oxidase and a Biosensor for Glucose Based on a Glass Carbon Electrode Modified with MoS2 Nanosheets Decorated with Gold Nanoparticles. Microchim Acta 2014, 181, 1497-1503.

9. $\quad$ Cui, H. F.; Zhang, K.; Zhang, Y. F.; Sun, Y. L.; Wang, J.; Zhang, W. D.; Luong, J. H. T. Immobilization of Glucose Oxidase into a Nanoporous TiO2 Film Layered on Metallophthalocyanine Modified Vertically-Aligned Carbon Nanotubes for Efficient Direct Electron Transfer. Biosens Bioelectron 2013, 46, 113-118.

10. Guiseppi-Elie, A.; Lei, C. H.; Baughman, R. H. Direct Electron Transfer of Glucose Oxidase on Carbon Nanotubes. Nanotechnology $2002,13,559-564$.

11. Vilian, A. T. E.; Chen, S. M. Direct Electrochemistry and Electrocatalysis of Glucose Oxidase Based Poly(L-arginine)-Multiwalled Carbon Nanotubes. Rsc Adv 2014, 4, 50771-50781.

12. Kamyabi, M. A.; Hajari, N.; Turner, A. P. F.; Tiwari, A. A High-Performance Glucose Biosensor Using Covalently Immobilised Glucose Oxidase on a Poly(2,6-diaminopyridine)/Carbon Nanotube Electrode. Talanta 2013, 116, 801-808.

13. Yu, Y. Y.; Chen, Z. G.; He, S. J.; Zhang, B. B.; Li, X. C.; Yao, M. C. Direct Electron Transfer of Glucose Oxidase and Biosensing for Glucose Based on PDDA-Capped Gold Nanoparticle Modified Graphene/Multi-Walled Carbon Nanotubes Electrode. Biosens Bioelectron 2014, 52, 147-152.

14. Wang, Y.; Li, H. X.; Kong, J. L. Facile Preparation of Mesocellular Graphene Foam for Direct Glucose Oxidase Electrochemistry and Sensitive Glucose Sensing. Sensor Actuat B-Chem 2014, 193, 708-714. 